\title{
Asymptotic Completeness for Multi-Particle Schroedinger Hamiltonians with Weak Potentials
}

\author{
Rafael Josf Iorio, Jr. and Michael O'Carroll \\ Departamento de Matemática, Pontificıa Universidade Católica, Rio de Janeiro. Brasil
}

Received March 15, 1972

\begin{abstract}
We show that the non-relativistic quantum mechanical $n$-body Hamiltonians $T(k)=T+k V$ and $T$, the free particle Hamiltonian, are unitarily equivalent in the center of mass system, i.e., $T(k)=W_{ \pm}(k) T W_{ \pm}(k)^{-1}$ for $k$ sufficiently small and real. $V=\sum_{i} V_{i}$, a sum of $\frac{n(n-1)}{2}$ real pair potentials, $V_{l}$, depending on the relative coordinate $x_{i} \in R^{3}$ of the pair $i$, where $V_{i}$ is required to behave like $\left|x_{i}\right|^{-2-\varepsilon}$ as $\left|x_{l}\right| \rightarrow \infty$ and like $\left|x_{i}\right|^{-2+\varepsilon}$ as $\left|x_{i}\right| \rightarrow 0 . T(k)$ is the self-adjoint operator associated with the form sum $T+k V$. There are no smoothness requirements imposed on the $V_{l}$. Furthermore $W_{ \pm}(k)=\underset{t \rightarrow \pm \infty}{s-\lim } e^{i T(k) t} e^{-\imath T t}$ are the wave operators of time dependent scattering theory and are unitary. This result gives a quantitative form of the intuitive argument based on the Heisenberg uncertainty principle that a certain minimum potential well depth and range is needed before a bound state can be formed. This is the best possible long range behavior in the sense that if $k V_{i} \leqq C_{i}\left|x_{i}\right|^{-b}$, $0<b \leqq 2$ for $\left|x_{i}\right|>R_{\imath}\left(0<R_{\imath}<\infty\right)$ and all $C_{i}$ are negative then $T(k)$ has discrete eigenvalues and $W_{ \pm}(k)$ are not unitary.
\end{abstract}

\section{Introduction}

In this article we treat the scattering and spectral problem for an $n$-body system in non-relativistic quantum mechanics with weak potentials. We show that the method of Kato [1] used to show asymptotic completeness and unitarity of the wave operators for weak potentials in the two-body case can be applied to obtain similar results in the $n$-body case. More precisely we show that in the center of mass system Hilbert space $H=L^{2}\left(R^{3 n-3}\right)$ the self-adjoint operators $T(k)=T+k V$ (the selfadjoint operator associated with a form sum) and $T$ (the free particle Hamiltonian) are unitarily equivalent for sufficiently small, real $k$. The potential $V=\sum_{i} V_{i}$ is a sum of pair potentials, $V_{i}$, which are real-valued measurable functions depending on the relative coordinates $x_{i} \in R^{3}$ of the pair $i$. Writing

$$
A_{i}=\left|V_{i}\right|^{1 / 2}, \quad B_{i}=\left(\operatorname{sign} V_{i}\right) A_{i},
$$

the result follows from the crucial fact that the operators $A_{i}(T-z)^{-1} B_{j}^{*}$ admit bounded analytic extensions for $\operatorname{Im} z \neq 0$, the bound being in- 
dependent of $z$ for $\operatorname{Im} z \neq 0$ and moreover that this is sufficient, i.e. compactness is not necessary.

The equivalence of $T$ and $T(k)$ is implemented by the unitary operators

$$
W_{ \pm}(k), \text { i.e., } \quad T(k)=W_{ \pm}(k) T W_{ \pm}(k)^{-1}
$$

where $W_{ \pm}(k)^{-1}=W_{ \pm}(k)^{*}$. The operators $W_{ \pm}(k)$ admit an absolutely convergent power series expansion in $k$. Pair potentials behaving like $1 /\left|x_{i}\right|^{2-\varepsilon}$ as $\left|x_{i}\right| \rightarrow 0$ and like $1 /\left|x_{i}\right|^{2+\varepsilon}$ as $\left|x_{i}\right| \rightarrow \infty$ are allowed $(\varepsilon>0)$ (see Theorem 1.1 for the precise conditions). There are no conditions of smoothness imposed on the potential functions. We also show that the operators

$$
\begin{aligned}
W_{ \pm}(k) & =\underset{t \rightarrow \pm \infty}{s-\lim } e^{i T(k) t} e^{-i T t} \\
W_{ \pm}(k)^{-1} & =\underset{t \rightarrow \pm \infty}{s-\lim _{t} e^{i T t} e^{-i T(k) t}=W_{ \pm}(k)^{*},}
\end{aligned}
$$

i.e., are the wave operators of time-dependent scattering theory. Thus the wave operators exist and their ranges are all $H$. This result of course also gives information about the spectrum of $T(k)$, in the center of mass system; namely, it is absolutely continuous, the singular continuous spectrum being absent. Thus there can be no point spectrum and we have a quantitative form of the heuristic argument using the Heisenberg uncertainty principle on the potential shape and well depth which will exclude bound states. Furthermore the long-range behavior of our result can not be improved. What is meant by this is that for pair potentials which fall off like $1 /\left|x_{i}\right|^{b}, 0<b \leqq 2$, for $\left|x_{i}\right| \rightarrow \infty$, no matter how small $k>0$ is there is an infinite point spectrum if all $V_{i}$ are negative. For a proof of this fact see Simon [2]. Thus the $W_{ \pm}(k)$ can not be unitary since the ranges of $W_{ \pm}(k)$ are orthogonal to the point spectrum of $T(k)$.

In Section I we introduce necessary notation and state our results. In Section II we give the proofs of the results.

For other results on $n$-body completeness in the general case see Hepp [3] and for repulsive potentials see Lavine [4]. For $n$-body spectral results see Simon [2], Balslev and Combes [5] and Albeverio [6].

\section{Results}

Throughout this paper we will maintain the notation of Kato [1]. We consider the $n$-particle self-adjoint Schroedinger Hamiltonian operator $T(k)$ which formally equals $T+k V$ where $T$ is the free particle self-adjoint center of mass kinetic energy operator and $V=\sum_{i} V_{i}$, the potential, is a sum of $n(n-1) / 2$ pair potentials $V_{i}$, such as $i=(l m)$, 
$l, m=1,2, \ldots, n(l<m)$. Each $V_{i}$ is a real-valued, measurable function depending only on the difference coordinates, $x_{i} \in R^{3}$, of the pair $i$ and $k$ is a real parameter. We write $V_{i}=B_{i}^{*} A_{i}$, where

$$
A_{i}=\left|V_{i}\right|^{1 / 2}, \quad B_{i}=\left(\operatorname{sign} V_{i}\right)\left|V_{i}\right|^{1 / 2} .
$$

The operators $T$ and $A_{i}, B_{i}$ are defined as maximum multiplication operators on the Fourier transform of $H=L^{2}\left(R^{3 n-3}\right)$ and $H$, respectively. Thus $T$ is self-adjoint and $A_{i}, B_{i}$ are closed.

In order that the theorem of Kato [1] be directly applicable we introduce the Hilbert space $H^{\prime}=H \oplus H \oplus \cdots \oplus H(n(n-1) / 2$ summands $)$ and let $V=B^{*} A$. The operators $A$ and $B$ are defined by

$X u=\left(X_{1} u, X_{2} u, \ldots, X_{\frac{n(n-1)}{2}} u\right)$ for $u \in D(X)=\bigcap_{i} D\left(X_{i}\right) \subset H, X=A(B)$, $X_{i}=A_{i}\left(B_{i}\right)$ and are closed linear operators as mappings from $D(X) \subset H$ to $H^{\prime}$. We point out however that it is not necessary to introduce $H^{\prime}$ and the results will still be valid but the Kato proof will then require modification. The resolvent of the self-adjoint operator $T$ will be denoted by $R(z)$.

We not state the crucial lemma from which our results will follow.

Lemma 1.1. The norm of the closure, $\left[C_{i} R(z) D_{j}\right]$, of the operator $C_{i} R(z) D_{j}$ for $\operatorname{Im} z \neq 0$ where $C_{i}, D_{i}=A_{i}, A_{i}^{*}, B_{i}, B_{i}^{*}$ has the majorization

$$
\begin{aligned}
\left\|\left[C_{i} R(z) D_{j}\right]\right\| \leqq & \left(2 m_{j} c_{i j}\right) /(4 \pi) \cdot\left(a^{-1}+b^{-1}\right) \\
& \cdot\left[\left\|V_{i}\right\|_{L^{p / 2}\left(R^{3}\right)}\left\|V_{j}\right\|_{L^{p / 2}\left(R^{3}\right)}\right]^{b 2^{-1}(a+b)^{-1}} \\
& \cdot\left[\left\|V_{i}\right\|_{L^{q / 2}\left(R^{3}\right)}\left\|V_{j}\right\|_{L^{q / 2}\left(R^{3}\right)}\right]^{2^{-1}(a+b)^{-1}},
\end{aligned}
$$

where $i, j$ are the same pair or not completely disjoint pairs. In (1.1) $a=-3 p^{-1}+1>0, b=3 q^{-1}-1>0,1 \leqq q<3<p \leqq \infty$ and $m_{j}^{-1}=m_{k}^{-1}$ $+m_{l}^{-1}$ with $j=(k l), k<l$. The constant

$$
c_{i j}=1 \quad \text { if } i=j \text { and } c_{i j}=\left(m_{k}+m_{l}\right) m_{k}^{-1} \text { for } k<l \text {, }
$$

$i=(l m), j=(k l) ; c_{i j}=\left(m_{k}+m_{l}\right) m_{l}^{-1}$ for $k<l, i=(k m), j=(k l)$. For $i, j$ completely disjoint pairs we have

$$
\begin{aligned}
\left\|\left[C_{i} R(z) D_{j}\right]\right\| & \leqq 2\left(\sup _{z}\left\|\left[A_{i} R(z) A_{i}\right]\right\|\right)^{1 / 2}\left(\sup _{z}\left\|\left[A_{j} R(z) A_{j}\right]\right\|\right)^{1 / 2} \\
& \leqq 2 \max _{i, j}\left(\sup _{z}\left\|\left[A_{i} R(z) A_{j}\right]\right\|\right)
\end{aligned}
$$

where the sup is taken over all $z, \operatorname{Im} z \neq 0$.

By specializing theorems 1.5, 3.9 and 4.1 of Kato [1] to our case and using the same notation as in Lemma 1.1 we have 
Theorem 1.1. For some constant $N<\infty$ let $V$ be such that

$$
\begin{aligned}
\max _{i j} 2(n(n-1) / 2) & {\left[\left(\frac{2 m_{j} c_{i j}}{4 \pi}\right)\left(a^{-1}+b^{-1}\right)\right] } \\
\cdot & {\left[\left\|V_{i}\right\|_{L^{p / 2}\left(R^{3}\right)}\left\|V_{j}\right\|_{L^{p / 2}\left(R^{3}\right)}\right]^{b 2^{-1}(a+b)^{-1}} } \\
\cdot & {\left[\left\|V_{i}\right\|_{L^{q / 2}\left(R^{3}\right)}\left\|V_{j}\right\|_{L^{q / 2}\left(R^{3}\right)}\right]^{a 2^{-1}(a+b)^{-1}}<N }
\end{aligned}
$$

(the factor 2 occurring after $\max$ in (1.3) can be dropped for $n=2$ or 3 ) then for any real $k$ with $|k|<1 / N$

a) $T(k)$ is the unique self-adjoint operator determined from the resolvent

$$
R(z, k)=R(z)-k\left[R(z) B^{*}\right](1+k Q(z))^{-1} A R(z)
$$

where $Q(z)=\left[A R(z) B^{*}\right]$ is a bounded operator from $H^{\prime}$ to $H^{\prime}$ and $\|Q(z)\|<N$ for $\operatorname{Im} z \neq 0$.

b) The operators $T$ and $T(k)$ are unitarily equivalent, i.e.

$$
T(k)=W_{ \pm}(k) T W_{ \pm}(k)^{*}
$$

where $W_{ \pm}(k)$ are unitary and are defined by

$$
\left(W_{ \pm} u, v\right)=(u, v) \mp(k / 2 \pi i) \int_{-\infty}^{+\infty}\left(A R(\lambda \pm i 0) u, B R(\lambda \mp i 0, k)^{*} v\right) d \lambda
$$

with $u, v \in H . W_{ \pm}(k), W_{ \pm}(k)^{*}$ admit absolutely convergent (in operator norm) series expansions in $k$.

c) The operators $W_{ \pm}(k), W_{ \pm}(k)^{*}$ of part $b$ are the wave operators of scattering theory, i.e. on all $H$ we have

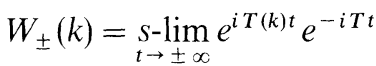

$$
\begin{aligned}
& W_{ \pm}(k)^{*}=\underset{t \rightarrow \pm \infty}{s-\lim _{t}} e^{i T t} e^{-i T(k) t}
\end{aligned}
$$

and the scattering operator $S=W_{+}(k)^{*} W_{-}(k)$ is unitary.

\section{Remarks}

1. $T(k)$ defined through (1.4) agrees with the definition of $T(k)$ defined by the quadratic forms method of Simon [7] as each $V_{i} \in L^{3 / 2}\left(R^{3}\right)$ by (1.3) and $L^{3 / 2}\left(R^{3}\right) \subset R$ (the Rollnik class).

2. In (1.5) $A R(\lambda \pm i 0) u$ are $H^{\prime}$ valued functions of $\lambda$ and exist a.e. for $-\infty<\lambda<\infty$ as boundary values of the analytic vector $A R(z) u$. Similar remarks apply to $B R(\lambda \mp i 0, k)^{*} v$.

3. For the case $n=2$ Kato [1] (see Theorem 6.1) showed that $V \in R$ is sufficient for the conclusions of Theorem 1.1 to hold for small $k$. 


\section{Proof of Lemma and Theorem}

Proof of Lemma 1.1. We will make estimates to establish sufficient conditions on the $V_{i}$ so that the operators

$$
C_{i} R(z) D_{j} \quad C_{i}, D_{j}=A_{i}, A_{i}^{*}, B_{i}, B_{i}^{*}
$$

admit norm bounded analytic extensions, $\left[C_{i} R(z) D_{j}\right]$, where the bound is uniform for $\operatorname{Im} z \neq 0$.

More precisely, we will show

$$
\begin{aligned}
& \sup \left\|\left[C_{i} R(z) D_{j}\right]\right\| \\
& <\left[\left(\frac{4 \pi}{2 m_{j}}\right)^{-3 p^{-1}} a_{i j}^{-3 p^{-1}+1}\left(-3 p^{-1}+1\right)^{-1}\left\|V_{i}\right\|_{L^{p / 2}\left(R^{3}\right)}^{1 / 2}\left\|V_{j}\right\|_{L^{p / 2}\left(R^{3}\right)}^{1 / 2}\left(c_{i j}\right)^{3 p-1}\right. \\
& \left.+\left(\frac{4 \pi}{2 m_{j}}\right)^{-3 q^{-1}} a_{i j}^{-3 q^{-1}+1}\left(3 q^{-1}-1\right)^{-1}\left\|V_{i}\right\|_{L^{q / 2}\left(R^{3}\right)}^{1 / 2}\left\|V_{j}\right\|_{L^{q / 2}\left(R^{3}\right)}^{1 / 2}\left(c_{i j}\right)^{3 q^{-1}}\right]
\end{aligned}
$$

where $a_{i j}>0,1 \leqq q<3<p \leqq \infty$ and $m_{j}^{-1}=m_{k}^{-1}+m_{l}^{-1}$ for $j=(k l), k<l$. The constant $c_{i j}=1$ if $i=j$ and $c_{i j}=\left(m_{k}+m_{l}\right) m_{k}^{-1}$ for $k<l, i=(l m)$, $j=(k l) ; c_{i j}=\left(m_{k}+m_{l}\right) m_{l}^{-1}$ for $k<l, i=(k m), j=(k l)$. The expression (2.2) holds for $i, j$ the same pair or not completely disjoint pairs. Minimizing the right hand side of (2.2) with respect to the $a_{i j}$ we have

$$
\begin{aligned}
\left.\sup _{z} \|\left[C_{i} R(z) D\right]\right] \| \leqq & \left(2 m_{j} c_{i j}\right)(4 \pi)^{-1} \cdot\left(a^{-1}+b^{-1}\right) \\
& \cdot\left[\left\|V_{i}\right\|_{L^{p / 2}\left(R^{3}\right)}\left\|V_{j}\right\|_{L^{p / 2}\left(R^{3}\right)}\right]^{b 2^{-1}(a+b)^{-1}} \\
& \cdot\left[\left\|V_{i}\right\|_{L^{a / 2}\left(R^{3}\right)}\left\|V_{j}\right\|_{L^{a / 2}\left(R^{3}\right)}\right]^{a 2^{-1}(a+b)^{-1}}
\end{aligned}
$$

where $a=-3 p^{-1}+1>0$ and $b=3 q^{-1}-1>0$.

For $i, j$ completely disjoint pairs we have

$$
\begin{aligned}
\sup _{z}\left\|\left[C_{i} R(z) D_{j}\right]\right\| & \leqq 2\left(\sup _{z}\left\|\left[C_{i} R(z) C_{i}\right]\right\|\right)^{1 / 2}\left(\sup _{z}\left\|\left[D_{j} R(z) D_{j}\right]\right\|\right)^{1 / 2} \\
& \leqq 2 \max _{i, j}\left(\sup _{z}\left\|\left[C_{i} R(z) D_{j}\right]\right\|\right) .
\end{aligned}
$$

We now make the estimates required to establish (2.2).

Since $A_{i}=A_{i}^{*} \geqq 0, B_{i}=A_{i} U_{i}=U_{i} A_{i}$ where $U_{i}$ is a partial isometry it is sufficient to bound the norms $\left\|A_{i} R(z) A_{j} u\right\|$ where

$$
u \in D\left(A_{j}\right)=D\left(A_{j}^{*}\right)=D\left(B_{j}\right)=D\left(B_{j}^{*}\right) .
$$

For the case of $i, j$ the same and $i, j$ different but not disjoint pairs we reduce the calculation to two body considerations and follow a method of Kato [1]. For $i, j$ disjoint pairs we reduce the problem to the case where the pairs are the same. 
Case 1. $i=j$, i.e., $i=(k l)$. We have

$$
\left\|A_{i} e^{-i T t} A_{i} u\right\|_{2}=\left\|A_{i} e^{-i T_{i} t} A_{i} u\right\|_{2}
$$

where $T_{i}$ is the relative kinetic energy of the pair $i$. We show that

$$
\left\|A_{i} e^{-i T_{i} t} A_{i} u\right\|_{2} \leqq\left(4 \pi t / 2 m_{i}\right)^{-3 p^{-1}}\left\|A_{i}\right\|_{L^{p}\left(R^{3}\right)}^{2}\|u\|_{2}, \quad u \in D\left(A_{i}\right), t>0
$$

where $m_{i}^{-1}=m_{k}^{-1}+m_{l}^{-1}$ so that, using the representation

$$
R(z) u=i \int_{0}^{\infty} e^{i z t} e^{-i T t} u d t, \quad \operatorname{Im} z>0
$$

we have for $\operatorname{Im} z>0$

$$
\begin{aligned}
\left\|A_{i} R(z) A_{i} u\right\|_{2} & \leqq \int_{0}^{\infty}\left\|A_{i} e^{-i T t} A_{i} u\right\|_{2} d t \\
& \leqq \int_{0}^{a_{1 i}}\left\|A_{i} e^{-i T_{i} t} A_{i} u\right\|_{2} d t+\int_{a_{11}}^{\infty}\left\|A_{i} e^{-i T_{i} t} A_{i} u\right\|_{2} d t .
\end{aligned}
$$

For $\operatorname{Im} z<0$ we obtain a similar majorization. We obtain (2.2) by substituting (2.5) with $p$ for the first integrand of (2.6) and (2.5) with $q$ replacing $p$ in the second integrand of (2.6). We now derive (2.5). Denote the coordinates by $\left(x, x_{R}\right)$ where $x \in R^{3}$ is the relative coordinate of the pair $i$ and $x_{R}$ are the other $3 n-6$ coordinates. We have for $t>0$

$$
\begin{aligned}
\left(e^{-i T_{i} t} A_{i} u\right) & \left(x, x_{R}\right) \\
= & \left(4 \pi i t / 2 m_{i}\right)^{-3 / 2} \int \exp \left[-2 m_{i}|x-y|^{2} / 4 i t\right] A_{i}(y) u\left(y, x_{R}\right) d y .
\end{aligned}
$$

The spatial integrations are understood to be over $R^{3}$ and are to be interpreted as limit in mean relations. With $v\left(x, x_{R}\right)=\exp \left(-2 m_{i}|x|^{2} / 4 i t\right)$ - $A_{i}(x) u\left(x, x_{R}\right)$ we have, using the Hausdorff-Young inequality [1],

$$
\left[\int\left|\left(e^{-i T_{\imath} t} A_{i} u\right)\left(x, x_{R}\right)\right|^{r^{\prime}} d x\right]^{1 / r^{\prime}} \leqq C_{i}\left(r^{\prime}, t\right)\left[\int\left|v\left(x, x_{R}\right)\right|^{r} d x\right]^{1 / r}
$$

with $r^{\prime-1}+r^{-1}=1, C_{i}\left(r^{\prime}, t\right)=\left(4 \pi t / 2 m_{i}\right)^{3\left(r^{-1}-2^{-1}\right)}$. But

$$
\left[\int\left|v\left(x, x_{R}\right)\right|^{r} d x\right]^{1 / r} \leqq\left\|A_{i}\right\|_{L^{2 p}\left(R^{3}\right)}\left[\int\left|u\left(x, x_{R}\right)\right|^{2} d x\right]^{1 / 2}
$$

with $r^{-1}=2^{-1}+(2 p)^{-1}$. Thus

$$
\begin{aligned}
\int\left|\left(A_{i} e^{-i T_{i} t} A_{i} u\right)\left(x, x_{R}\right)\right|^{2} d x d x_{R} \leqq & \int\left[\int\left|A_{i}(x)\right|^{2 s} d x\right]^{1 / s} \\
\cdot & {\left[\int\left|\left(e^{-i T_{i} t} A_{i} u\right)\left(x, x_{R}\right)\right|^{2 s^{\prime}} d x\right]^{1 / s^{\prime}} d x_{R} }
\end{aligned}
$$

with $s^{-1}+s^{\prime-1}=1,2 s^{\prime}=r^{\prime}$, which upon substituting (2.8) and (2.9) in (2.10) gives (2.5).

Case 2. $i \neq j$ but $i$ and $j$ not disjoint pairs. It is sufficient and for definiteness we consider $i=(23), j=(12)$. In analogy with case $1,(2.4)$, we 
have

$$
\left\|A_{23} e^{-i T t} A_{12} u^{\prime}\right\| \leqq\left\|A_{23} e^{-i T_{j} t} A_{12} u\right\|, \quad u=e^{-i\left(T-T_{J}\right) t} u^{\prime}
$$

where in this case $T_{j}$ is the relative kinetic energy of the pair $j$. The relative coordinates of 1 and 2 we denote by $y_{1} \in R^{3}$ and $y_{1}$ plays the role of the variable $x$ of case 1 . We denote the coordinates by $\left(y_{1}, x_{R}\right)$. We have with $\left\|A_{12} u\left(\cdot, x_{R}\right)\right\|_{L^{r}\left(R^{3} \cdot d v_{1}\right)}<\infty$,

$$
v\left(y_{1}, x_{R}\right)=e^{-\left|y_{1}\right|^{2} 2 m_{J} / 4 i t}\left(A_{12} u\right)\left(y_{1}, x_{R}\right)
$$

$m_{j}^{-1}=m_{1}^{-1}+m_{2}^{-1}$, and from the Hausdorff-Young inequality

$$
\left\|e^{-i T_{J} t} A_{12} u\left(, x_{R}\right)\right\|_{L^{r}\left(R^{3}, d y_{1}\right)} \leqq C_{j}\left(r^{\prime}, t\right)\left\|v\left(, x_{R}\right)\right\|_{L^{r}\left(R^{3}, d y_{1}\right)}
$$

where $r^{\prime-1}+r^{-1}=1$. Then

$$
\left\|A_{23} e^{-i T_{J} t} A_{12} u\right\|_{2}^{2}=\int\left|A_{23}\right|^{2}\left|\left(e^{-i T_{J} t} A_{12} u\right)\left(y_{1}, x_{R}\right)\right|^{2} d y_{1} d x_{R} .
$$

We use the Holder inequality in $y_{1}$ with $s^{-1}+s^{-1}=1$ to obtain

$$
\begin{aligned}
\left\|A_{23} e^{-i T_{J} t} A_{12} u\right\|_{2}^{2} \leqq & \int\left[\int\left|A_{23}\right|^{2 s} d y_{1}\right]^{1 / s}\left[\int \mid\left(e^{-i T_{j} t} A_{12} u\right)\right. \\
& \left.\left.\cdot\left(y_{1}, x_{R}\right)\right|^{2 s^{\prime}} d y_{1}\right]^{1 / s^{\prime}} d x_{R} \\
\leqq & \left(m_{12} / m_{1}\right)^{3 / s}\left\|A_{23}\right\|_{L^{2 s}\left(R^{3}\right)}^{2} C_{j}\left(r^{\prime}, t\right)^{2} \\
& \cdot \int\left\|v\left(, x_{R}\right)\right\|_{L^{r}\left(R^{3}, d y_{1}\right)}^{2} d x_{R}
\end{aligned}
$$

with $r^{\prime}=2 s^{\prime}$. In arriving at (2.15) we have used the fact that $A_{23}\left(x_{2}^{\prime}-x_{3}^{\prime}\right)$ $=A_{23}\left(m_{1} m_{12}^{-1} y_{1}+\sum_{i} b_{i} x_{R}^{i}\right), x_{2}^{\prime}, x_{3}^{\prime}$ denote the coordinates of 2 and 3 , $m_{12}=m_{1}+m_{2}$, and where $\sum_{i} b_{i} x_{R}^{i}$ is a linear combination of the other $x_{R}$ coordinates (see Appendix 1 of Simon [2]). Thus we have

$$
\left.\left.\left|\int\right| A_{23}\right|^{2 s} d y_{1}\right|^{1 / s}=\left(m_{12} / m_{1}\right)^{3 / s}\left\|A_{23}\right\|_{L^{2 s}\left(R^{3}\right)}^{2} .
$$

In the case where $A_{13}$ occurs in place of $A_{23}$ we have $A_{13}\left(x_{1}^{\prime}-x_{3}^{\prime}\right)=A_{13}$ $\left(m_{2} m_{12}^{-1} y_{1}+\sum_{i} c_{i} x_{R}^{i}\right)$. Using the Holder inequality again in (2.15) we obtain

$$
\begin{aligned}
\left\|v\left(, x_{R}\right)\right\|_{L^{2}\left(R^{3}, d y_{1}\right)}^{2} & =\left[\int\left|A_{12} u\left(y_{1}, x_{R}\right)\right|^{r} d y_{1}\right]^{2 / r} \\
& \leqq\left[\int\left|A_{12}\right|^{r k} d y_{1}\right]^{2 / r k}\left[\int\left|u\left(y_{1}, x_{R}\right)\right|^{r k^{\prime}} d y_{1}\right]^{2 / r k^{\prime}}
\end{aligned}
$$

where $k^{-1}+k^{\prime-1}=1$. Let $r k^{\prime}=2$ then

$$
\left\|A_{23} e^{-i T_{j} t} A_{12} u\right\|_{2}^{2} \leqq\left(m_{12} / m_{1}\right)^{6 / q}\left\|A_{23}\right\|_{L^{q}\left(R^{3}\right)}^{2}\left\|A_{12}\right\|_{L^{q}\left(R^{3}\right)}^{2} C^{2}\left(r^{\prime}, t\right)\|u\|_{2}^{2}
$$

with $q=2 r(2-r)^{-1}$ or as $\left\|u^{\prime}\right\|_{2}=\|u\|_{2}$ we have

$$
\begin{aligned}
& \left\|A_{23} e^{-i T t} A_{12} u^{\prime}\right\|_{2} \\
& \leqq\left(m_{12} / m_{1}\right)^{3 / q}\left\|A_{23}\right\|_{L^{q}\left(R^{3}\right)}\left\|A_{12}\right\|_{L^{q}\left(R^{3}\right)}\left(4 \pi t / 2 m_{j}\right)^{-3 / q}\left\|u^{\prime}\right\|_{2}
\end{aligned}
$$


which is analogous to (2.5). Proceeding in the same manner as from (2.5) to (2.6) we obtain (2.2) for this case.

Case 3. $i \neq j$ with the pair $i$ disjoint from the pair $j$. We have for $\operatorname{Im} z>0$ with the sup taken over $v \in D\left(A_{j}\right),\|v\|=1$,

$$
\begin{aligned}
\left\|A_{i} R(z) A_{j} u\right\| & \leqq \sup \left|\int_{0}^{\infty}\left(v, A_{i} e^{i T t} A_{j} u\right) d t\right| \\
& =\sup \left|\int_{0}^{\infty}\left(e^{i T, t} v, A_{i} e^{-i\left(T-T_{j}\right) t} A_{j} u\right) d t\right| \\
& =\sup \left|\int_{0}^{\infty}\left(A_{j}^{*} e^{i T_{j} t} v, A_{i} e^{-i\left(T-T_{j}\right) t} u\right) d t\right| \\
& \leqq\left(\sup \left(\int_{0}^{\infty}\left\|A_{j}^{*} e^{i T_{j} t} v\right\|^{2} d t\right)^{1 / 2}\right)\left(\int_{0}^{\infty}\left\|A_{i} e^{-i T_{i} t} u\right\|^{2} d t\right)^{1 / 2}
\end{aligned}
$$

where $T_{i}, T_{j}$ are the relative kinetic energies of the pairs $i, j$ respectively. From Lemma 3.6, Eq. (3.9) and Theorem 5.1, Eq. (5.3) of Kato [1] we have (recalling that $A_{k}: D\left(A_{k}\right) \subset H \rightarrow H$ )

$$
\begin{gathered}
\int_{-\infty}^{\infty}\left\|A_{k} e^{-i t T_{k}} w\right\|^{2} d t \leqq 2\|w\|^{2} \sup \left\|A_{k} R\left(z, T_{k}\right) A_{k}^{*} u^{\prime}\right\| /\left\|u^{\prime}\right\| \\
\operatorname{Im} z \neq 0, \quad u^{\prime} \in D\left(A_{k}^{*}\right), \quad u^{\prime} \neq 0
\end{gathered}
$$

for $k=i$ or $j, w \in H$ so that

$$
\begin{aligned}
\left\|A_{i} R(z) A_{j} u\right\| \leqq & 2\left[\sup \left\|A_{i} R\left(z, T_{i}\right) A_{i}^{*} v^{\prime}\right\| /\left\|v^{\prime}\right\|\right]^{1 / 2} \\
& \cdot\left[\sup \left\|A_{j} R\left(z, T_{j}\right) A_{j}^{*} u^{\prime}\right\| /\left\|u^{\prime}\right\|\right]^{1 / 2}\|u\|
\end{aligned}
$$

where the sup in (2.21) is taken over $\operatorname{Im} z \neq 0, v^{\prime} \in D\left(A_{i}^{*}\right), u^{\prime} \in D\left(A_{j}^{*}\right)$, $v^{\prime} \neq 0, u^{\prime} \neq 0$. The resolvents $R\left(z, T_{m}\right)$ in (2.21) are the resolvents of the operators $T_{m}$. By noting that each term in the brackets of (2.21) just reduces to the type given by (2.6) we arrive at (2.3).

Proof of Theorem 1.1. Since $T$ is self-adjoint it is obvious that all the hypotheses of Theorems 1.5, 3.9 and 4.1 of Kato [1] are satisfied except for the $T$-smoothness [1, Definition 1.2] of $A$ and $B$ and that

$$
\left\|A R(z) B^{*} u\right\| \leqq N\|u\|, \quad \operatorname{Im} z \neq 0, \quad u \in D\left(B^{*}\right) \subset H^{\prime} .
$$

Thus our proof consists in showing that these two hypotheses are satisfied. From Remark 1.10 of Kato [1] we see that $T$-smoothness of $A_{i}$ and $B_{i}$ imply that $A$ and $B$ are $T$-smooth. From the hypothesis (1.3) we see (referring to Lemma 1.1) that $\left\|\left[A_{i} R(z) A_{i}\right]\right\|$ is bounded uniformly for $\operatorname{Im} z \neq 0$. Since $A_{i}=A_{i}^{*} \geqq 0, B_{i}=A_{i} U_{i}=U_{i} A_{i}$ this implies the $T$-smoothness of $A_{\imath}$ and $B_{\imath}$ using Theorem 5.1, Eq. (5.3) of Kato [1]. 
Eq. (2.22) is valid since

$$
\begin{aligned}
\left\|A R(z) B^{*} u\right\| & \leqq \sup _{\|v\|=1}\left|\left(v, A R(z) B^{*} u\right)\right| \\
& \leqq \sup _{\|v\|=1}\left\{\sum_{i j} \mid\left(v_{i}, A_{i} R(z) B_{j}^{*} u_{j}\right) \|\right. \\
& \leqq \sup _{\|v\|}\left[\left(\sum_{i j}\left\|v_{i}\right\|^{2}\left\|u_{j}\right\|^{2}\right)^{1 / 2}\left(\sum_{i j}\left\|\left[A_{i} R(z) B_{j}^{*}\right]\right\|^{2}\right)^{1 / 2}\right\} \\
& \leqq \max _{i, j}\left\|\left[A_{i} R(z) B_{j}^{*}\right]\right\|(n(n-1) / 2)\|u\|
\end{aligned}
$$

and by the hypotheses (1.3) the right-hand side of (2.23) is less than $N\|u\|$ for $\operatorname{Im} z \neq 0$.

\section{References}

1. Kato, T.: Wave Operators and Similarity for Some Non-selfadjoint Operators. Math. Ann. 162, 258-279 (1966).

2. Simon, B.: On the Infinitude or Finiteness of the Number of Bound States of an N-Body Quantum System, I. Helv. Phys. Acta 43, (6) 607--630 (1970).

3. Hepp, K.: On the Quantum Mechanical N-Body Problem. Helv. Phys. Acta 42, (3) $425-458(1969)$.

4. Lavine, R.: Commutators and Scattering Theory. I. Repulsive Interactions. Commun. math. Phys. 21, 301-323 (1971).

5. Balslev, E., Combes, J. M.: Spectral Properties of Many-body Schroedinger Operators with Dilatation-analytic Interactions. Commun. math. Phys. 22, 280-294 (1971).

6. Albeverio, S.: (to appear in Ann. Phy.).

7. Simon, B.: Quantum Mechanics for Hamiltonians defined as Quadratic Forms. Princeton, New Jersey: Princeton University Press. 1971.

\section{R. J. Iorio, Jr.}

Departamento de Matemática Ponificia Universidade Católica

Rio de Janeiro, Brasil 